\title{
Situación del sistema de vigilancia de casos de Dengue en un municipio de Colombia
}

\author{
The status of the dengue surveillance system in a Colombian \\ municipality
}

\author{
Diego Zea ${ }^{1}$ y Lyda Osorio ${ }^{2}$
}

\begin{abstract}
1 Centro Internacional de Entrenamiento e Investigaciones Médicas (CIDEIM). dfz77@yahoo.com 2 Universidad del Valle, Facultad de Salud, Escuela de Salud Pública, Grupo de Epidemiología y Salud Poblacional (GESP) lyda1oso@gmail.com
\end{abstract}

Recibido 21 Enero 2011/Enviado para Modificación 3 Octubre 2011/Aceptado 18 Octubre 2011

\section{RESUMEN}

Objetivos La vigilancia del dengue es fundamental para definir, implementar y evaluar las intervenciones y para la detección precoz de epidemias. Las direcciones municipales de salud son el nodo que garantiza la calidad y cobertura del sistema y los usuarios inmediatos de la información. El objetivo del presente estudio fue evaluar el sistema de vigilancia de dengue en una dirección municipal de salud en el Valle del Cauca en el año 2008.

Métodos Se siguió el modelo del CDC con énfasis en 1) la descripción del sistema, 2) los recursos disponibles, 3) el cumplimiento en la notificación y ajuste de casos y 4) el uso de la información. Se realizaron entrevistas a funcionarios y revisión de los datos de dengue de la Secretaria Departamental y Municipal de Salud y del Laboratorio Departamental de Salud Pública.

Resultados La cobertura de notificación fue del 91,3\%, se evidenciaron falta de uso del sistema electrónico y retraso en el envío de los datos. Tres personas estaban a cargo de todas las funciones de salud pública pero no tenían formación en sus respectivas áreas ni conocimiento de los protocolos. No se realizaba análisis sistemático de los datos.

Conclusiones Como en otros municipios de Colombia, la falta de capacitación y perfil de los funcionarios y de otros recursos, y el alto recambio de personal son los aspectos básicos más débiles del sistema de vigilancia en salud pública del municipio. Se han implementado correctivos tendientes a mejorar el sistema de vigilancia en el municipio.

Palabras Clave: Dengue, vigilancia epidemiológica, salud pública, Colombia (fuente: DeCS, BIREME)

\section{ABSTRACT}

Objective The surveillance of dengue cases is essential for defining, implementing and assessing interventions and promptly detecting outbreaks. Municipal health 
authorities are the key stakeholders guaranteeing surveillance system quality and coverage and are the first users of the information so collected. The present study was aimed at assessing how well the dengue case surveillance system was operating in a municipality in the Valle del Cauca department of Colombia during 2008.

Methods The CDC proposed model was used. Emphasis was placed on describing system operation, the available resources, compliance with reports and case adjustment and using information. Interviews were held with health workers. Dengue surveillance system data from the municipality, departmental health authorities and the Valle del Cauca Public Health Laboratory were reviewed.

Results Notification coverage was $91.3 \%$. A lack of using surveillance software and delays in notification were identified. Three people were in charge of all public health responsibilities and none of them had had any training or prior knowledge regarding public health surveillance protocols. There had not been any systematic data analysis. Conclusions As in other areas of Colombia, the lack of training, professional profile and other resources as well as a high turnover of public health personnel were the weakest points of the surveillance system in the municipality. Corrective measures for improving the surveillance system have now been implemented in the municipality which was studied.

Key Words: Dengue, epidemiological surveillance, public health, Colombia (source: $\mathrm{MeSH}, \mathrm{NLM}$ ).

$\mathrm{E}$ 1 Dengue es un problema de salud pública que afecta aproximadamente 3 billones de personas que viven, principalmente, en las zonas urbanas de 102 países tropicales y subtropicales. Se estima que en el mundo se presentan anualmente 100 millones de casos de fiebre dengue, 500000 de dengue grave y 25000 muertes (1). En América, el problema ha ido creciendo debido a la diseminación del principal vector, Aedes aegypti, en el continente y la circulación de los 4 serotipos del virus dengue $(1,2)$. Para el periodo de 2001 a 2005, Brasil (1 852 330) y Colombia (243 019) fueron los países de la región que más casos de dengue reportaron y a su vez Colombia aportó el 70 $\%(155 / 221)$ del total de muertes atribuidas a dengue para el mismo periodo en toda América (3). En el 2010, Colombia presentó la mayor epidemia de dengue en los últimos años con más de 150000 casos reportados, de los cuales $6 \%$ (9 393) fueron dengue grave y 210 muertes confirmadas (4).

El control del dengue y la reducción de la mortalidad atribuible a esta causa hacen parte de las metas de Colombia para el logro de los Objetivos de Desarrollo del Milenio (5). Para el control del dengue y la evaluación del logro de estas metas es fundamental tener un sistema de vigilancia que genere información útil para orientar la definición, implementación y evaluación de las intervenciones que se realizan y detectar oportunamente la presencia de epidemias. En Colombia, la vigilancia epidemiológica del dengue, junto con 
otros 81 eventos de interés en salud pública, se realiza a través del Sistema Nacional de Vigilancia en Salud Pública (SIVIGILA), el cual se rige por el decreto 3518 del 2006(5). El proceso de vigilancia de dengue inicia con la notificación a través de una ficha individual que diligencia un profesional de salud en la institución donde se identificó el caso. El flujo de información va entonces de las Unidades Primarias Generadoras de Datos (UPGDs) hacia las direcciones municipales de salud y de allí a las direcciones departamentales, quienes a su vez la envían al Instituto Nacional de Salud y de éste al Ministerio de la Protección Social y los organismos internacionales (Figura 1). De acuerdo con la norma, la ficha individual se debe enviar semanalmente a la dirección municipal de salud en medio físico o a través de archivos electrónicos (.txt) generados en un programa de computador denominado igualmente SIVIGILA. La versión más reciente del programa de computador SIVIGILA, a la fecha del estudio, era la del 2008 y se implementó a partir de enero de 2009. Los detalles operativos para la notificación de casos de dengue se encuentran en el protocolo de vigilancia elaborado por el Instituto Nacional de Salud (7). Si bien prácticamente todo el sistema de salud, tanto público como privado, es responsable de la implementación y desarrollo del sistema de vigilancia, las direcciones municipales de salud son el nodo que garantiza la calidad y cobertura del sistema y los usuarios inmediatos de la información generada. Para esto, las direcciones municipales de salud deben: 1) garantizar la infraestructura y el talento humano necesario para la gestión de la vigilancia, 2) organizar y coordinar la red de vigilancia de su jurisdicción, 3) realizar la gestión interinstitucional e intersectorial para la implementación y desarrollo de acciones de vigilancia y garantizar el flujo continuo de información, 4) organizar la comunidad para lograr la participación de la misma en la realización de actividades propias de la vigilancia en salud pública, 5) realizar la búsqueda activa de casos y contactos cuando se requiera, 6) investigar las epidemias y 7) realizar el análisis de la situación de salud en su jurisdicción (8).

Identificar cómo las direcciones de salud responden ante estos compromisos es útil para que las autoridades de salud departamentales y nacionales ofrezcan el respaldo y acompañamiento necesarios que garanticen el cumplimiento de los objetivos del sistema de vigilancia. Con el fin identificar las dificultades que pueden estar presentando los municipios en el país para realizar adecuadamente el proceso de Vigilancia en Salud Pública y en este caso en particular, la vigilancia de casos de dengue, se analizó la situación del sistema de vigilancia de dengue en una Unidad Notificadora Municipal en el Valle del Cauca en el 2008. 
Figura 1. Flujo de información en el Sistema de Vigilancia en Salud Pública de Colombia

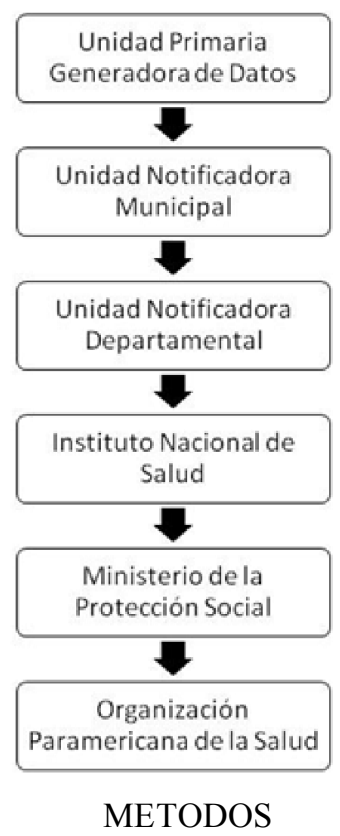

De acuerdo con su población e ingresos corrientes de libre destinación (Ley 617 de 2000), el municipio de estudio se clasifica como de tercera categoría. Esta categoría le confiere, por un lado, autonomía presupuestal y, por otro, la responsabilidad directa de las acciones de vigilancia y control de los eventos de interés en salud pública, incluido el dengue. Presenta una alta proporción de dengue grave (entre 10 y $36 \%$ del total de dengue reportado) y cuenta con la circulación de los dos vectores implicados en la transmisión de la enfermedad (Aedes aegypti y Ae. albopictus) y de los cuatro serotipos del virus (DENV1-4). Durante el periodo comprendido entre el año 2000 y el 2007 se reportaron 4648 casos de dengue, de los cuales 900 (19\%) correspondieron a dengue grave. El año con mayor número de casos de dengue fue 2002, con 1298 casos. Se omite el nombre y referencias del municipio, con el fin de proteger la confidencialidad de la información. El presente estudio fue aprobado por el Comité de Ética de Investigación del CIDEIM y la Universidad del Valle. 
Se realizó un análisis de tipo descriptivo usando métodos cuantitativos y cualitativos. Para el análisis del sistema de vigilancia de dengue se tomó como guía el modelo propuesto por el CDC para evaluar sistemas de vigilancia en Salud Pública (9). Debido a limitaciones de personal para realizar la evaluación de todos los atributos del sistema se decidió enfocar en los componentes del sistema que dependen directamente de la Unidad Notificadora Municipal que fue el sujeto central del estudio. Estos fueron: 1. La descripción de la operación del sistema; 2 . Los recursos disponibles; 3 . El cumplimiento en la notificación y ajuste de casos, y; 4. El uso de la información. La recolección de la información se realizó a través de entrevistas no estructuradas de los investigadores al Secretario de Salud y los funcionarios encargados de Salud Pública, Estadística y Saneamiento Ambiental, revisión de los informes, fichas de notificación en físico y archivos planos, y observación directa. Los ejes temáticos de las entrevistas giraron en torno al manejo y uso de la información de dengue y los recursos disponibles para el sistema de vigilancia. Uno de los investigadores recibió capacitación en el programa SIVIGILA 2008 (10).

Para la operación del sistema se describió el flujo de la información y los componentes del sistema que incluyó el número total de UPGDs con los códigos individuales e información necesaria para su configuración en el SIVIGILA 2008 y los procedimientos para el manejo de la información. En los recursos disponibles se tuvo en cuenta el número, perfil, tipo de contratación y conocimiento de los lineamientos de vigilancia en salud pública de los funcionarios. También se observaron las instalaciones y las herramientas utilizadas para la vigilancia epidemiológica del dengue. El cumplimiento en la notificación se evaluó con base en los reportes de envío que registra el SIVIGILA y el ajuste de casos con base en el indicador de casos confirmados por laboratorio. Los resultados de pruebas de IgM realizadas a las muestras enviadas por el municipio en estudio durante el año 2008 se obtuvieron del Laboratorio Departamental de Salud Pública. Con respecto al uso de la información se determinaron los siguientes aspectos: proceso de retroalimentación de información a las entidades de salud, el contenido de los informes trimestrales obligatorios de vigilancia de dengue enviados por el municipio a la Secretaría Departamental de Salud y las medidas de control tomadas con base en la información de vigilancia.

Finalmente, se ingresaron a SIVIGILA 2008 los datos correspondientes a dengue del municipio notificados en el 2008. Para ello se utilizaron los archivos planos en formato SIVIGILA 2007 generados por las UPGDs y también fichas epidemiológicas físicas enviadas por las UPGDs que no contaban con el programa SIVIGILA. Una de las UPGDs notificó 42 casos en un formato antiguo por lo 
que no contaban con la información correspondiente a hospitalización, de modo que se asumieron como hospitalizados los casos de dengue grave $(\mathrm{n}=15)$ y como no hospitalizados los casos de dengue no grave $(n=27)$.

El registro de códigos de barrios, veredas y corregimientos se obtuvo de la Secretaría de Planeación Municipal y fue necesario agregar el código a todos los registros individuales de los casos de dengue, pues sólo aparecía la dirección del caso o el nombre del barrio o vereda. Para el análisis de la información de dengue se utilizó el programa SIVIGILA 2008 y se calcularon los indicadores para dengue establecidos por el Instituto Nacional de Salud (7). El análisis de las entrevistas fue manual para la descripción de todos los hallazgos.

\section{RESULTADOS}

Operación del sistema y recursos disponibles

El sistema de vigilancia epidemiológica para todos los eventos de salud pública del municipio estaba conformado por tres unidades: estadística, saneamiento y salud pública. Cada una de ellas con un funcionario a cargo. Por lo tanto, el sistema de vigilancia de casos de dengue contaba con tres funcionarios, además del secretario de salud. Dos de los empleados trabajaban por nómina y el empleado de estadística por contratación de nueve meses, lo cual implicaba que la Secretaría de Salud quedaba sin apoyo estadístico durante tres meses al año. El funcionario de salud pública y el de estadística no tenían formación profesional o técnica en sus respectivas áreas de trabajo. Cada una de las tres unidades se ubicaba en diferentes niveles del edificio. La unidad de estadística, a diferencia de las otras dos, no contaba con impresora ni existía la facilidad de impresión en red. El funcionario de salud pública y el de estadística desconocían los lineamientos de vigilancia en salud pública y los protocolos de vigilancia de dengue y demás eventos de interés en salud pública.

Cumplimiento en la notificación y ajuste de casos

El municipio contaba con 23 UPGDs de las cuales 21 (91,3\%) enviaron sus reportes a la Secretaría de Salud Municipal. Seis eran laboratorios clínicos, 2 hospitales públicos de nivel II de atención y las demás eran clínicas privadas. Dos de las UPGDs no tenían código y subíndice de caracterización, el cual es necesario para notificar en archivos planos. El 61\% (n=14) notificaban a través de archivos planos utilizando la versión más actualizada (2008) del programa SIVIGILA, de éstas sólo una lo hizo de manera continua durante las 53 semanas epidemiológicas de 2008. Siete (30,4 \%) UPGDs reportaron por medio físico a través de fichas de notificación porque no tenían instalado el SIVIGILA. 
En cuanto a la prontitud de la notificación, existía retraso de un día puesto que las UPGDs notificaban a la Secretaría de Salud Municipal los días martes (en vez del día lunes) y ésta lo hacía los días miércoles (en vez del martes) a la Secretaría Departamental de Salud. No se realizaba ajuste de casos ya que no existía comunicación de los resultados de las muestras procesadas para IgM dengue entre el Laboratorio Departamental de Salud Pública y la Secretaria Municipal de Salud. Por otro lado, las UPGDs de la red pública tampoco informaban los resultados a la Secretaría Municipal. De igual manera sucedió con las UPGDs del régimen contributivo y sus laboratorios.

Análisis y uso de la información

El funcionamiento del sistema utilizaba un flujo bidireccional de la información entre cada uno de sus componentes, una vez los datos ingresan a la Secretaría. Inicialmente la Unidad de Estadística recibía los datos en forma de archivos planos y/o fichas individuales de notificación enviadas por las UPGDs. A pesar de recibir los archivos planos no se hacía uso de ellos pues no existía conocimiento de su finalidad y manera de ser utilizados. La Secretaría de Salud Municipal no contaba con el software SIVIGILA versión 2008 y los funcionarios no estaban capacitados para manejar este programa o versiones anteriores. Una vez llegaban a la unidad de estadística, las fichas epidemiológicas eran enviadas a la unidad de saneamiento para que se realizara "filtro" de acuerdo al grado de severidad de la trombocitopenia o a la presencia de hemorragias reportadas. De este modo se seleccionaban las fichas notificadas como dengue no grave que tenían plaquetas en descenso o cuyo único reporte era bajo (el funcionario no especificó el valor) para realizar visitas de campo con fines de control vectorial. Posteriormente, se avisaba a la unidad de salud pública para que, a través de ésta, se alertara de estos casos a las instituciones notificadoras con el fin de hacer seguimiento clínico y paraclínico. En caso de presentarse un "alto" número de casos de dengue, la unidad de salud pública se encargaba de informar a los hospitales para prepararse en forma logística para la atención de los casos. No se elaboraban canales endémicos pero se informó que en las situaciones de epidemia, se desarrollaron campañas educativas a la comunidad, para evitar la proliferación de criaderos en los domicilios.

$\mathrm{Al}$ revisar los informes trimestrales enviados a la Secretaría Departamental de Salud se encontró que solamente contenían el total de casos y la periodicidad de las acciones de control vectorial. No se incluían los indicadores, canales endémicos y distribuciones poblacionales y geográficas de la enfermedad y tampoco se concluía acerca de la problemática de la enfermedad en el municipio. 
Situación de dengue

De acuerdo con la información recopilada, durante el 2008 se presentaron 67 casos de dengue en el municipio, de los cuales 55 (82\%) correspondieron a dengue no grave y $12(18 \%)$ a dengue grave. El $60 \%$ de los casos fueron hombres; sin embargo, la distribución para dengue grave fue homogénea en ambos sexos (50\%). El grupo de edad con mayor número de casos fue el comprendido entre 15 y 44 años con $30(44,7 \%)$ mientras que el grupo de 5 a 14 años de edad ( 5 casos) fue el más afectado para dengue grave.

Se hospitalizaron $20(30 \%)$ personas por dengue, de quienes 11 correspondieron a dengue clásico y 9 a dengue hemorrágico. Esto reflejó un alto porcentaje de hospitalización por dengue no grave $(55 \%)$ con respecto a dengue grave (45\%). Del total de hospitalizados, 14 (70 \%) eran hombres. El grupo de edad con mayor número de hospitalizaciones fue el de menores de 15 años con $11(55 \%)$ casos.

De acuerdo a la información consignada en medio físico y archivos planos de SIVIGILA 2008, se encontró que 8 (66 \%) casos de dengue aparecen como confirmados al momento de ser notificados a pesar de no tener registro del resultado de la prueba de IgM para dengue. De acuerdo a la distribución geográfica, de las 6 comunas que posee la cabecera municipal, la comuna más afectada fue la uno, con 16 casos $(24 \%)$. Cuatro casos $(6 \%)$ fueron procedentes de la zona rural y en 10 casos $(15 \%)$ no se registró procedencia.

Los indicadores calculados para el año 2008 fueron: 1 . Incidencia de dengue: 5,8 por 100000 habitantes; 2. Porcentaje de letalidad por dengue y porcentaje de muertes por dengue: cero; 3. Porcentaje de casos probables de dengue grave: $33 \%$.

\section{DISCUSIÓN}

Con el objetivo de identificar los retos que las secretarías municipales de salud enfrentan para el cumplimiento de sus funciones dentro del sistema de vigilancia de dengue, este estudio analizó la situación del sistema de vigilancia de dengue en una Unidad Notificadora Municipal del Valle del Cauca.

La falta de capacitación y perfil de los funcionarios son los aspectos básicos más débiles del sistema de vigilancia en salud pública del municipio. El conocimiento acerca de sus funciones, los lineamientos del sistema de vigilancia en salud pública y los protocolos de vigilancia de los eventos de interés en salud pública, resulta definitivo para trazar el rumbo del sistema y para establecer los objetivos 
primordiales de acción frente al evento dengue en el municipio. El desarrollo de manuales que expliquen en forma breve y clara las funciones y los procedimientos clave de los cargos de salud pública, epidemiología, estadística y aquellos relacionados con la vigilancia en salud pública podría contribuir en la capacitación de los funcionarios, cuyo alto recambio dificulta la sostenibilidad de las iniciativas de capacitación que lideran las secretarias departamentales de salud y el Instituto Nacional de Salud. Sin embargo, estos manuales requieren el acompañamiento para resolver dudas y motivar su uso. En este sentido, desarrollamos e implementamos un manual de vigilancia epidemiológica para dengue en el municipio de estudio. El manual se ha constituido en un documento de referencia y consulta pero su uso requirió un acompañamiento continuo y procesos de capacitación persona a persona. Contratar y mantener personal capacitado en las funciones de vigilancia en dengue y los eventos de interés en salud pública es el primer reto para las direcciones municipales de salud.

La no utilización del software establecido por el Instituto Nacional de Salud (SIVIGILA 2008), para el proceso de notificación, empobrece el flujo y la calidad de la información del sistema de vigilancia en salud pública. Personal capacitado en sus funciones tendría como prioridad mantenerse actualizado en los lineamientos y herramientas necesarias para llevar a cabo la vigilancia epidemiológica de dengue. A través del acompañamiento ofrecido se logró la implementación de SIVIGILA 2008 y su uso rutinario. Sin embargo, aun se requiere soporte para brindar asesoría y resolver dudas.

Si bien el conocimiento es indispensable para llevar a cabo las funciones de vigilancia de dengue, es claro que la carga laboral de los funcionarios desborda su capacidad de respuesta. Los tres funcionarios tenían bajo su responsabilidad no sólo la vigilancia y control de dengue sino de todos los más de 80 eventos de interés en salud pública y acciones de contingencia. A esta falta de recursos humanos se suma la falta de recursos físicos como facilidades de impresión. Este factor afecta no solo a dengue sino a todos los eventos objeto de vigilancia en salud pública. De acuerdo con Lopez y col. esta misma situación se evidenció en 11 municipios de 5 Departamentos del país en el año 2004, lo cual sugiere que ha habido poco progreso en mejorar la capacidad de los municipios para cumplir con sus funciones de vigilancia en salud pública (11). En respuesta a esta situación el municipio contrató un epidemiólogo para apoyar las funciones de salud pública. Ante la falta de recursos, se hace necesario que el Instituto Nacional de Salud lidere un proceso que permita priorizar las acciones de vigilancia en salud pública. 
Otros retos se relacionan con la necesidad de establecer canales de comunicación eficientes al interior de la Secretaría Municipal y con otras entidades oficiales como por ejemplo entre los laboratorios involucrados en el procesamiento de las muestras de IgM para dengue y la Secretaría de Salud Municipal, lo cual se requiere para realizar el ajuste de casos. Esta situación ha mejorado a través del envío periódico de los resultados de IgM a través del correo electrónico. Sin embargo, se requiere acompañamiento permanente a las UPGDs para que los ajustes se hagan efectivos.

La situación de dengue en el municipio durante 2008 refleja el cumplimiento de la meta nacional (incidencia $<10 / 100000$ habitantes); sin embargo, este estudio no realizó evaluación de la sensibilidad del sistema y es factible que se encuentre subregistro. De hecho, en dengue el subregistro es común y algunos autores proponen que se requiere vigilancia activa (12). La vigilancia activa demanda una mayor capacidad en personal de los municipios o las UPGDs lo cual impactaría la capacidad de respuesta. La notificación por parte de las UPGD del municipio durante 2008, a pesar de estar cercana al $100 \%$ (91,3 \%), está por debajo de la meta nacional al evaluarla semanalmente.

A raíz de la publicación, por parte de la OMS, de las guías de dengue (13), Colombia adoptó, en marzo de 2010, nuevas definiciones de caso cambiando la anterior clasificación de dengue clásico, dengue hemorrágico y choque por dengue con y sin signos de alarma y dengue grave (14). Este cambio y la alerta generada por la situación de epidemia en el 2010 que afrontaron la mayoría de Departamentos del país (4), podrían constituirse en la oportunidad para implementar estrategias que garanticen los recursos y entrenamiento necesarios para fortalecer la vigilancia de dengue en las Unidades Notificadoras Municipales.

En conclusión, se evidenciaron deficiencias en la operación del sistema de vigilancia de dengue, la disponibilidad de recursos y probablemente como consecuencia de éstas, deficiencias en oportunidad y calidad de la notificación y el uso de la información. Se considera necesario realizar evaluaciones del sistema de vigilancia no sólo de dengue sino de otros eventos de interés en salud pública, para conocer la problemática que enfrentan, implementar mejorías y así poder estimar la situación real de los eventos de interés en salud pública del país

Agradecimientos: A todos los funcionarios de la dirección municipal de salud por su participación en el estudio y suministro de la información. Este estudio fue financiado por COLCIENCIAS contrato No. 2229-408-20422. 


\section{REFERENCIAS}

1. World Health Organization. Prevention and control of dengue and dengue haemorrhagic fever, Comprehensive guidelines. New Delhi: World Health Organization Regional publication, SEARO No 29; 1999.

2. Guzmán MC, Kourí G. Dengue: An update. Lancet Infect Dis. 2002;2:33-42.

3. Panamerican Health Organization. Health in the Americas 2007. Vol. 1. Washington D.C.: PAHO Scientific and Technical publication $\mathrm{N}^{\circ} 622.2007$ p. 101-107.

4. Instituto Nacional de Salud. Subdirección de Vigilanciay Control de Salud Pública. Boletín epidemiológico semanal. Disponible en http://new.paho.org/col/index2.php?option=com_content\&do_pdf= 1\&id=923 Fecha de acceso Ene 20 de 2011.

5. Departamento Nacional de Planeación. Consejo Nacional de Política Económica y Social. República de Colombia. Metas y Estrategias de Colombia para el Logro de los Objetivos de Desarrollo del Milenio 2015. Documento Conpes Social 91. Bogotá 14 de marzo de 2005.

6. Ministerio de la Protección Social de Colombia. Decreto Número 3518 de 2006. Sistema de vigilancia en salud pública. 2006; p 1-10.

7. Instituto Nacional de Salud de Colombia. Vigilancia y control en salud pública, SIVIGILA 2008: Protocolo de vigilancia de dengue. Instituto nacional de salud. 2007.

8. Instituto Nacional de Salud. Lineamientos para el Sistema de Vigilancia y Control en Salud Pública 2007. Unidades Notificadoras Municipales. Sistema de Vigilancia en Salud Pública. Instituto Nacional de Salud.

9. Centers for Disease Control and Prevention. Guidelines working group. Updated guidelines for evaluating public health surveillance systems. MMWR Recomm Rep 2001;27;50(RR13): $1-35$.

10. Instituto Nacional de Salud. Vigilancia y control en salud pública, SIVIGILA 2008: Manual del Usuario. Instituto Nacional de salud. 2008.

11. López Y, González C, Gallego BN, Moreno AL. Rectoría de la vigilancia en salud pública en el sistema de seguridad social en salud de Colombia: estudio de casos Biomédica 2009;29:567-81.

12. Gubler DJ. Active surveillance for dengue and dengue hemorrhagic fever. Bol Oficina Sanit Panam. 1989;107(1):22-30.

13. World Health Organization (WHO) and the Special Programme for Research and Training in Tropical Diseases (TDR). Dengue: guidelines for diagnosis, treatment, prevention and control - New edition. 2009 WHO/HTM/NTD/DEN/2009.

14. Instituto Nacional de Salud. Grupo de vigilancia y control de enfermedades transmisibles (Grupo de enfermedades transmitidas por vectores). Protocolo de vigilancia y control del dengue. 25 septiembre de 2009 INT-R02.002.4020-009. 